\title{
Assessment of Cytotoxicity and Genotoxicity in Patients with Rheumatoid Arthritis and its Relation to Disease Activity and Medications
}

\author{
Sally S. Tayel ${ }^{1}$, Manal Y. Tayel ${ }^{1}$, Hanan S. Mahrous ${ }^{2}$, Eiman A. Soliman ${ }^{1}$ \\ Departments of Internal Medicine ${ }^{1}$, Faculty of Medicine, \\ Human Genetics ${ }^{2}$, Medical Research Institute; Alexandria University; Egypt
}

\begin{abstract}
Objectives: To assess the chromosomal aberrations (CAs) as a measure of cytotoxicity and micronuclei (MNi) and nucleoplasmic bridges (NPB) as a measure of genotoxicity in patients with rheumatoid arthritis (RA) and its relation to the disease activity. The work also aimed to assess the possible cytotoxicity and genotoxicity induced by some Disease Modifying Anti-Rheumatic Drugs (DMARDs) used for treatment of RA patients. Patients and Methods: The study included 20 female RA patients treated with DMARDs (MTX and SSZ), 10 RA patients not receiving DMARDs (used NDAIDs), and 10 control. Peripheral blood samples were taken to assess the cytotoxicity by chromosomal analysis (karyotyping) using solid Giemsa stain and GTG- banding according to the International System for Human Chromosome Nomenclature. Genotoxicity and DNA damage was assessed by the miconucleus (MN) test using cytochalasin-B. Results: The DMARDs RA patients had a mean age of $42.2 \pm 11.76 \mathrm{yr}$ and a mean disease duration of $7.95 \pm 6.43 \mathrm{yr}$. The MTX dose was $50 \mathrm{mg} / \mathrm{wk}$ and the SSZ dose was $500 \mathrm{mg} / 8$ hours, and the mean duration of use was $7.1 \pm 6.27 \mathrm{yr}$. The $20 \mathrm{RA}$ patients of the DMARDs group received folic acid dose of $0.5 \mathrm{mg} /$ day for the same period as DMARDs. The mean age of the non- MTX RA (NSAIDs) group was $43.1 \pm 13.5 \mathrm{yr}$, the mean disease duration was $4.3 \pm 1.9 \mathrm{yr}$. The results showed a high significant increase $(\mathrm{p}<0.001)$ of chromosomal breaks, satellite association, chromosome endoreduplication, aneuploidy, and other CAs in RA patients compared to control. This significant difference was not correlated with disease activity. Cytotoxicity of MTX was illustrated by the significant increase in the number of satellite association, and cells with aneuploidy when compared to the NSAIDs group. The frequency of micronuclei (MNi), nucleoplasmic bridges (NPB), and necrotic and/or apoptotic cells were significantly higher in RA patients when compared to control $(\mathrm{p}<0.05)$ but not correlated to disease activity. RA patients receiving DMARDs showed a significant increase in the number of binucleated cells with one MN when compared to the NSAIDs group. Conclusion: CAs, MNi, and NPB were increased RA patients so suggesting that cytotoxicity and genotoxicity are manifestations of RA disease itself. These CAs, MNi, and NPB were not correlated to the degree of disease activity, and seemed to be randomly distributed except in the case of X-chromosome anomalies. MTX is still cyto-and genotoxic to RA patients despite the associated use of folic acid. This toxicity might be due to the high dose and its long time of use in the present study. [Egypt J Rheumatology \& Clinical Immunology, 2016; 4(1): 67-80]
\end{abstract}

\section{INTRODUCTION}

Rheumatoid arthritis (RA) is the most common autoimmune inflammatory joint disease worldwide ${ }^{1}$. It affects $1 \%$ of the world population and triggers joint inflammations that may worsen patient's quality of life $^{2}$.

Despite the noticeable advances in the treatment of RA, mortality among patients with RA continues higher than that of the general population, with no significant changes in recent years ${ }^{3}$. Nowadays, efficient DMARDs, such as Methotrexate (MTX), as well as targeted immunomodulating agents, are available to relieve patients ${ }^{2}$.
For decades, the possible pathogenic effects of one or more disease-associated chromosomal regions (loci) have been investigated separately. These studies often led to controversies as some groups were able to confirm linkage of a certain single nucleotide polymorphism (SNP) to RA, while others could not ${ }^{4}$.

The association of certain chromosome aberrations with arthropathy has been described, but there are a limited number of reports in the literature ${ }^{5}$. Czako et al. ${ }^{6}$ mentioned that Juvenile rheumatoid arthritis has occasionally been reported in patients with deletion of $18 \mathrm{p}$, and thus most likely loss of a gene or genes on $18 p$ is responsible for various immunologic disorders occurring in these patients. 
A recent study performed by Maxwell et al. ${ }^{7}$ in (2012) has identified 6q23 as an important susceptibility locus for rheumatoid arthritis (RA). In the study performed by Orozco et al. ${ }^{8}$ in 2014 , a total of 3,223 UK RA cases and 5,272 UK controls were available for GWAS using 1,831,729 singlenucleotide polymorphisms (SNPs). This study identified a new RA locus mapping to 22q12.

Methotrexate (MTX) is a central agent in RA therapy, and is used either alone or in combination with biological DMARDs. However, a large proportion of RA patients $(20 \%-40 \%)$ either do not respond to or are unable to tolerate $\mathrm{MTX}^{9}$. At least 36 deaths have been attributed to bone marrow cytotoxicity associated with low dose MTX $^{10}$.

Suarez et al. ${ }^{11}$ have cited that salazopyrin (sulfasalazine; SASP) has become a common second line drug (DMARD) for the treatment of RA. Linares et al. ${ }^{12}$ mentioned that a high incidence of side effects limits therapy with SASP as a drug commonly used in the treatment of inflammatory bowel diseases such as ulcerative colitis, Crohn's disease and RA.

Genotoxicity describes a deleterious action on a cell's genetic material affecting its integrity ${ }^{13}$. The standard micronucleus test can provide the following measures of genotoxicity and cytotoxicity: Chromosome breakage, chromosome loss, chromosome rearrangement, cell division inhibition, necrosis and apoptosis ${ }^{14}$.

The aim of the present work was to assess the chromosomal aberrations (as a measure of cytotoxicity) and micronuclei (as a measure of genotoxicity) in patients with rheumatoid arthritis and its relation to the disease activity. The present work also aimed to assess the possible cytotoxicity and genotoxicity induced by some Disease Modifying Anti-Rheumatic Drugs (DMARDs) used for treatment of rheumatoid arthritis patients.

\section{SUBJECTS AND METHODS}

The study included 30 female patients and 10 control. The thirty female patients with rheumatoid arthritis were divided into two subgroups: Ten patients with RA receiving no medication except Non Steroidal Anti-Inflammatory Drugs (NSAIDs) and twenty patients with RA receiving DMARDs (Methotrexate, Azathioprine and Salazopyrin) as a single drug or in combination for at least 3 months period. Patients were recruited from the Rheumatology Department, Main Alexandria University Hospital. They fulfilled the new ACR/EULAR criteria for the diagnosis of the RA disease $^{15}$. Written consents from all patients were taken. Chromosomal analysis and the micronucleus test (cyto- and genotoxicity assessment) were performed at Alexandria Regional Centre for Women's health and Development. The study also included ten healthy female control subjects matching the same age.

All patients were subjected to the following: 1History taking as regards: a. Demographic data: Age, ethnic group (Nationality), smoking, any other associated disease (which might cause cyto- or genotoxicity as bilharziasis or cancer), and duration of the disease. b. Drug history: Type, dose, and duration of use of the DMARDs medication, and the use of folic acid with its dose. c. Pregnancy history: Use of contraceptive pills and number of abortions. 2Clinical data for the disease activity: This was done by applying the $4^{\text {th }}$ version of DAS28 (ESR or CRP) ${ }^{16} .3-$ Cytotoxicity assessment: It was performed by karyotyping (chromosomal analysis) for the study of numerical chromosomal aberrations (as aneuploidy; hypoploidy or hyperploidy) and structural chromosomal aberrations (as breaks, gaps, centric fusion, fragile sites, satellite associations, endoreduplication). Karyotyping ${ }^{17}$ was performed on peripheral blood samples using solid Giemsa stain and GTG- banding technique according to the International System for Human Chromosome Nomenclature ISCN $^{18}$. 4- The micronucleus test ${ }^{19}$ was performed using cytochalasin $\mathrm{B}$ to measure genotoxicity and DNA damage.

\section{Statistical Analysis}

Data were fed to the computer using IBM SPSS software statistical package version 20.0. Both statistical analysis and tabulation were done according to Altman ${ }^{20}$. Differences between 2 continuous variables were compared with student unpaired " $t$ " test. Differences between 3 or more continuous variables were compared with one way analysis of variance (ANOVA) using Scheffe's method for multiple comparisons. Differences between proportions were compared with the chi- squared test and Fisher's exact test. Level of significance was set at $\mathrm{p}<0.05^{15}$.

\section{RESULTS}

I. Results of the clinical data (Table 1):

Age of the control group ranged between 39-68 yr with a mean of $49.8 \pm 9.72$. Age of RA patients ranged between $15-75 \mathrm{yr}$ with a mean of $42.47 \pm 11.56$. Disease duration ranged between 1-30 yr with a mean of 6.73 \pm 5.77 . All cases of the DMARDs group $(n o=20)$ received folic acid with a dose of $0.5 \mathrm{mg} /$ day. Disease activity as judged by DAS28 ranged between 3.32-7.34 with a mean of $4.98 \pm 0.94$. Sixteen cases 
(53.3\%) had moderate disease activity while high disease activity was observed in $14(46.7 \%)$ cases. All cases were rheumatoid factor positive. Eighteen cases $(60 \%)$ with RA were on or have taken oral contraceptive pills (OCPs) for more than 5 years. Twenty one RA patients $(75 \%)$ out of 28 who had children have confirmed less severity of the RA disease during pregnancy while the answers were inconclusive in the other 7 patients. Breast feeding for more than a year was also associated with less disease activity in 17 cases $(81 \%)$ out of the 21 cases who had children and breast feeding. All cases were of low socioeconomic status, and 28 of them $(93.3 \%)$ were without university degree. Eleven cases $(36.7 \%)$ had abortions, 8 of them $(72.7 \%)$ were receiving DMARDs. All cases were Egyptian non-smokers

\section{Results of cytotoxicity:}

Table (2) and Figure (1) compare between chromosomal aberrations in RA patients and the control group. There was a significant difference in the chromosomal aberrations (CAs) between the two groups $(\mathrm{p}<0.05)$ and this difference was highly significant as regarding breaks $(\mathrm{p}<0.001)$. This finding indicates that CAs (as a measure of cytotoxicity) is a manifestation of the RA disease itself. There was no significant difference between the two groups as regards the MI

Table (3) illustrates the significance of CAs (as regards breaks, Sat Ass, dmin, endoreduplication, aneuploidy, and other aberrations; $\mathrm{p}<0.05$ ) between the control and the different groups of RA patients where statistically significant difference was found between the control and the NSAIDs group (p1), between the control and the DMARDs group (p2), between the control and the moderate disease activity group (p3), between the control and the high disease activity group (p4), and between the control and the 30 RA patients of the study (with moderate and high activity; p5) indicating that cytotoxicity is a part of the RA disease. There was neither significant difference between chromosomal aberrations encountered in the NSAIDs and the DMARDs group ( $\mathrm{p} 6$ where $\mathrm{p}>0.05$ ), nor between RA patients of moderate and high disease activity ( $\mathrm{p} 7$ where $\mathrm{p}>0.05$ ). No significant difference was observed between control and RA patients regarding the MI.

Table (4) shows the difference in CAs between RA patients on NSAIDs and DMARDs patients on MTX and MTX \& SSZ. There was a significant difference $(\mathrm{p}<0.05)$ between RA patients on MTX and those on NSAIDs as regards Sat Ass, endoreduplication and aneuploidy only (p1) while the difference in the other CAs as breaks, dmin, and other aberrations were not statistically significant $(p>0.05)$. This finding points to the toxicity of MTX which has been partially prevented by the use of folic acid in the patients of the present study. The same findings were found in the MTX \& SSZ group when compared to NSAIDs (p2) so denoting that no more toxicity was observed due to the use of SSZ. There was no significant difference between the MTX group and the MTX \& SSZ one which indicates whether that SSZ is not toxic or that its toxicity has been masked by the use of folic acid.

Table (5) shows the frequency of cells with CAs observed in the control, RA patients with disease durations between $1-<5$ years, $5-<10$ years, and 10-30 years where there was a highly significant difference $(\mathrm{P} \leq 0.001)$ in all the studied markers of cytotoxicity between the control and RA patients irrespective of the disease duration.

Table (6) demonstrates the frequency of CAs observed in the control, and in RA patients receiving DMARDs for a period of 1-5 years, and > 5 years. The results show a high significant difference between the control and RA patients on DMARDs irrespective of the time of use ( $\mathrm{p} 1$ and $\mathrm{p} 2$ where $\mathrm{p} \leq 0.001)$. RA patients who where on DMARDs for a longer duration (> 5 years) showed a statistically significant increase in all the cytogenetic markers (except other chromosomal aberrations; $\mathrm{p} 3$ was $\leq 0.05$ ).

\section{Results of genotoxicity (Micronucleus test):}

Table (7) and Figure (2) show that the difference between binucleated cells (BN) with $\mathrm{MNi}$ and $\mathrm{BN}$ cells with NPB and the necrotic and/or apoptotic cells between the 30 RA patients and the control group was statistically significant so illustrating that genotoxicity is a part of RA disease itself.

Table (8) shows that the number of $\mathrm{MNi}$ in the 30 RA patients was high in $86.67 \%$ ( $>6 \mathrm{MNi}$ ), and moderate in $13.33 \%(3-5 \mathrm{MNi})$ as compared to the control group where $80 \%$ had the normal number of MNi (0-2). This difference in the MNi number was highly significant $(<0.0001)$ so documenting the genotoxicity of RA.

Table (9) demonstrates that there was a significant difference in the BN cells with $\mathrm{MNi}, \mathrm{NPB}$, and necrotic and/or apoptotic cells between the control group and each of the following: the NSAIDs group (p1), the DMARDs group (p2), the moderate disease activity group (p3), the high disease activity group (p4), and the 30 RA patients with moderate and high disease activity (p5) where the $\mathrm{p}$ value was $<0.05$. There was no significant difference between NSAIDs and DMARDs groups except in the number of BN with one $\mathrm{MNi}$ (p6). There was also no significant difference between the moderate and high disease activity groups as regarding the genotoxicity ( $\mathrm{p} 7$ where $\mathrm{p}$ was $>0.05$ ). 
There was a significant difference in $\mathrm{BN}$ cells with one $\mathrm{MNi}$, NPB and necrotic and/or apoptotic cells between the NSAIDs group and the MTX one which agrees with the results of the cytotoxicity (chromosomal aberrations) and also between the NSAIDs and the MTX \& SSZ group. There was no significant difference between the MTX and the MTX \& SSZ groups (Table 10).

Table 1. Summary of the clinical data of the study groups.

\begin{tabular}{|c|c|c|c|c|c|c|}
\hline & & $\begin{array}{l}\text { Total cases } \\
\quad(\mathbf{n}=\mathbf{3 0})\end{array}$ & $\begin{array}{c}\text { NSAIDs } \\
(\mathrm{n}=10)\end{array}$ & $\begin{array}{c}\text { MTX } \\
(n=12)\end{array}$ & $\underset{\substack{\text { MTX,SSZ } \\
(\mathbf{n}=8)}}{\text { MTX }}$ & $\begin{array}{c}\text { DMARDs } \\
(\text { Total }(\text { no=20) }\end{array}$ \\
\hline \multirow{2}{*}{$\begin{array}{l}\text { Age } \\
(\mathrm{yr})\end{array}$} & Range & $15-75$ & $28-75$ & $29-63$ & $15-55$ & $15-63$ \\
\hline & Mean \pm SD & $42.47 \pm 11.56$ & $43.1 \pm 13.5$ & $43.08 \pm 13.45$ & $40.8 \pm 11.6$ & $42.2 \pm 11.76$ \\
\hline \multirow{2}{*}{$\begin{array}{c}\text { Disease Duration } \\
(\mathrm{yr})\end{array}$} & Range & $1-30$ & $1-7$ & $1-30$ & $3-14$ & $1-30$ \\
\hline & Mean \pm SD & $6.73 \pm 5.77$ & $4.3 \pm 1.9$ & $7.92 \pm 8.08$ & $8.0 \pm 4.2$ & $7.95 \pm 6.43$ \\
\hline \multirow{2}{*}{$\begin{array}{l}\text { Period of drug } \\
\text { Use (yr) }\end{array}$} & Range & $1-30$ & $1-6$ & $1-30$ & $2-14$ & $1-30$ \\
\hline & Mean \pm SD & $5.9 \pm 5.71$ & $3.5 \pm 2.72$ & $7.3 \pm 8.05$ & $6.8 \pm 4.2$ & $7.1 \pm 6.27$ \\
\hline \multirow{2}{*}{ Folic acid } & No. & 20 & 0 & 12 & 8 & 20 \\
\hline & $\%$ & 66.7 & 0.0 & 60 & 40 & 100 \\
\hline \multirow{2}{*}{ DAS28 } & Range & $3.0-7.3$ & $3.74-5.81$ & $3.32-6.64$ & $4.18-7.34$ & $3.32-7.34$ \\
\hline & Mean \pm SD & $4.98 \pm 0.94$ & $4.65 \pm 0.7$ & $5.0 \pm 0.99$ & $5.37 \pm 1.1$ & $5.15 \pm 0.86$ \\
\hline \multirow{2}{*}{$\begin{array}{c}\text { Disease activity } \\
\text { Moderate }\end{array}$} & No. & 16 & 7 & 6 & 3 & 9 \\
\hline & $\%$ & 53.3 & 43.8 & 37.5 & 18.7 & 56.2 \\
\hline \multirow{2}{*}{$\begin{array}{c}\text { Disease activity } \\
\text { High }\end{array}$} & No. & 14 & 3 & 6 & 5 & 11 \\
\hline & $\%$ & 46.7 & 21.4 & 42.9 & 35.7 & 78.6 \\
\hline \multicolumn{7}{|l|}{ Abortion } \\
\hline \multirow{2}{*}{0} & No. & 19 & 7 & 7 & 5 & 12 \\
\hline & $\%$ & 63.3 & 36.8 & 36.8 & 26.3 & 63.2 \\
\hline \multirow{2}{*}{1} & No. & 6 & 2 & 3 & 1 & 4 \\
\hline & $\%$ & 20 & 33.3 & 50.0 & 16.7 & 66.7 \\
\hline \multirow{2}{*}{2} & No. & 3 & 1 & 0 & 2 & 2 \\
\hline & $\%$ & 10 & 33.3 & 0.0 & 66.7 & 66.7 \\
\hline \multirow{2}{*}{ More (3-7) } & No. & 2 & 0 & 2 & 0 & 2 \\
\hline & $\%$ & 6.7 & 0.0 & 100.0 & 0.0 & 100 \\
\hline \multirow{2}{*}{$\begin{array}{l}\text { Total cases of } \\
\text { abortions }\end{array}$} & No. & 11 & 3 & 5 & 3 & 8 \\
\hline & $\%$ & 36.7 & 27.3 & 45.5 & 27.3 & 72.7 \\
\hline
\end{tabular}

Table 2. Comparison between the chromosomal aberrations in rheumatoid arthritis patients and the control group.

\begin{tabular}{|c|c|c|c|c|c|c|c|c|}
\hline & & Breaks & Sat Ass. & dmin & $\begin{array}{l}\text { Endo- } \\
\text { redup. }\end{array}$ & Aneupl. & $\begin{array}{c}\text { Other } \\
\text { Aberr. }\end{array}$ & MI \\
\hline $\begin{array}{c}\text { Control } \\
(10)\end{array}$ & $\begin{array}{c}\text { Range } \\
\text { Mean } \pm \text { SD }\end{array}$ & $\begin{array}{c}0-3 \\
0.6 \pm 1.07 \\
\end{array}$ & $\begin{array}{c}0-21 \\
6.2 \pm 6.49\end{array}$ & $\begin{array}{c}0.0-0.0 \\
0.0 \pm 0.0\end{array}$ & $\begin{array}{c}0.0-0.0 \\
0.0 \pm 0.0\end{array}$ & $\begin{array}{c}0.0-0.0 \\
0.0 \pm 0.0\end{array}$ & $\begin{array}{c}0.0-0.0 \\
0.0 \pm 0.0\end{array}$ & $\begin{array}{l}4.2-10.6 \\
6.8 \pm 2.11\end{array}$ \\
\hline $\begin{array}{l}\text { RA patients } \\
\text { (30) }\end{array}$ & $\begin{array}{c}\text { Range } \\
\text { Mean } \pm \text { SD }\end{array}$ & $\begin{array}{c}0-43 \\
14.77 \pm 11.58\end{array}$ & $\begin{array}{c}15-68 \\
38.16 \pm 14.77\end{array}$ & $\begin{array}{c}0-20 \\
2.03 \pm 4.77\end{array}$ & $\begin{array}{c}0-36 \\
9.7 \pm 8.31\end{array}$ & $\begin{array}{c}0-13 \\
5.3 \pm 4.5\end{array}$ & $\begin{array}{c}0-4 \\
0.70 \pm 0.51\end{array}$ & $\begin{array}{c}1.5-13.5 \\
5.76 \pm 3.25\end{array}$ \\
\hline P-value & & $<0.001^{*}$ & $<0.05^{*}$ & $<0.05^{*}$ & $<0.05^{*}$ & $<0.05^{*}$ & $<0.05^{*}$ & $>0.05$ N.S \\
\hline Endo-redup: & loreduplica & & Aneup: Ane & $\begin{array}{l}\text { inute } \\
\text { oidy }\end{array}$ & & r: Aberra & & \\
\hline
\end{tabular}


Tayel, et al.: Cytotoxicity and Genotoxicity in Patients with Rheumatoid Arthritis

Table 3. Frequency of cells showing chromosomal aberrations in the control, RA patients on NSAIDs, RA patients on DMARDs, RA patients with moderate and high disease activity (out of 100 cells/ case).

\begin{tabular}{|c|c|c|c|c|c|c|c|c|}
\hline & & Breaks & $\begin{array}{c}\text { Satellite } \\
\text { association }\end{array}$ & $\begin{array}{l}\text { Double } \\
\text { minutes }\end{array}$ & $\begin{array}{c}\text { Endo- } \\
\text { reduplication } \\
\end{array}$ & Aneuploidy & $\begin{array}{c}\text { Other } \\
\text { aberrations }\end{array}$ & Mitotic index \\
\hline \multirow{2}{*}{ Control (10) } & Range & $0-3$ & $0-21.0$ & $0.0-0.0$ & $0.0-0.0$ & $0.0-0.0$ & $0.0-0.0$ & $4.2-10.6$ \\
\hline & Mean \pm SD & $0.6 \pm 1.07$ & $6.2 \pm 6.49$ & $0.0 \pm 0.0$ & $0.0 \pm 0.0$ & $0.0 \pm 0.0$ & $0.0 \pm 0.0$ & $6.8 \pm 2.11$ \\
\hline \multirow{2}{*}{$\begin{array}{l}\text { RA patients on } \\
\text { NSAIDs (10) }\end{array}$} & Range & $0-36$ & $15-48$ & $0-15$ & $0-16$ & $0-8$ & $0-2$ & $1.5-10.1$ \\
\hline & Mean \pm SD & $13.0 \pm 12.53$ & $28.7 \pm 12.78$ & $3.0 \pm 5.37$ & $5.6 \pm 5.83$ & $2.1 \pm 2.96$ & $0.4 \pm 0.699$ & $5.72 \pm 3.02$ \\
\hline \multirow{2}{*}{$\begin{array}{l}\text { RA patients on } \\
\text { DMARDs (20) }\end{array}$} & Range & $0-43$ & $22-68$ & $0-20$ & $0-36$ & $0-13$ & $0-4$ & $2.4-13.5$ \\
\hline & Mean \pm SD & $15.7 \pm 11.3$ & $42.9 \pm 13.6$ & $1.55 \pm 4.5$ & $11.75 \pm 8.7$ & $6.9 \pm 4.3$ & $0.85 \pm 0.915$ & $6.07 \pm 3.21$ \\
\hline \multirow{2}{*}{$\begin{array}{c}\text { RA patients with } \\
\text { moderate disease } \\
\text { activity (16) }\end{array}$} & Range & $0-30$ & $15-68$ & $0-20$ & $0-36$ & $0-13$ & $0-4$ & $1.5-11.3$ \\
\hline & Mean \pm SD & $12.8 \pm 9.6$ & $34.38 \pm 15.6$ & $1.44 \pm 5.01$ & $9.56 \pm 8.8$ & $5.25 \pm 4.7$ & $0.69 \pm 0.48$ & $6.37 \pm 3.07$ \\
\hline \multirow{2}{*}{$\begin{array}{l}\text { RA patients with } \\
\text { high disease activity } \\
\text { (14) }\end{array}$} & Range & $0-43$ & $20-67$ & $0-15$ & $0-28$ & $0-11$ & $0-3$ & $2.4-13.5$ \\
\hline & Mean \pm SD & $17.0 \pm 13.5$ & $42.5 \pm 12.9$ & $2.71 \pm 4.56$ & $9.86 \pm 8.0$ & $5.36 \pm 4.5$ & $0.71 \pm 0.425$ & $5.48 \pm 3.18$ \\
\hline \multirow{2}{*}{$\begin{array}{l}\text { RA patients with } \\
\text { moderate and high } \\
\text { disease activity (30) }\end{array}$} & Range & $0-43$ & $15-68$ & $0-20$ & $0-36$ & $0-13$ & $0-4$ & $1.5-13.5$ \\
\hline & Mean \pm SD & $14.77 \pm 11.58$ & $38.16 \pm 14.77$ & $2.03 \pm 4.77$ & $9.7 \pm 8.31$ & $5.3 \pm 4.50$ & $0.7 \pm 0.51$ & $5.76 \pm 3.25$ \\
\hline $\mathrm{P}_{1}$ & & $<0.05^{*}$ & $<0.05^{*}$ & $<0.05^{*}$ & $<0.05^{*}$ & $<0.05^{*}$ & $<0.05^{*}$ & $>0.05$ N.S. \\
\hline $\mathrm{P}_{2}$ & & $<0.05^{*}$ & $<0.05^{*}$ & $<0.05^{*}$ & $<0.05^{*}$ & $<0.05^{*}$ & $<0.05^{*}$ & $>0.05$ N.S. \\
\hline $\mathrm{P}_{3}$ & & $<0.05^{*}$ & $<0.05^{*}$ & $<0.05^{*}$ & $<0.05^{*}$ & $<0.05^{*}$ & $<0.05^{*}$ & $>0.05$ N.S. \\
\hline $\mathrm{P}_{4}$ & & $<0.05^{*}$ & $<0.05^{*}$ & $<0.05^{*}$ & $<0.05^{*}$ & $<0.05^{*}$ & $<0.05^{*}$ & $>0.05$ N.S. \\
\hline $\mathrm{P}_{5}$ & & $<0.05^{*}$ & $<0.05^{*}$ & $<0.05^{*}$ & $<0.05^{*}$ & $<0.05^{*}$ & $<0.05^{*}$ & $>0.05$ N.S. \\
\hline $\mathrm{P}_{6}$ & & $>0.05$ N.S. & $>0.05$ & $>0.05$ & $>0.05$ & $>0.05$ & $>0.05$ & $>0.05$ \\
\hline P7 & & $>0.05$ N.S. & $>0.05$ & $>0.05$ & $>0.05$ & $>0.05$ & $>0.05$ & $>0.05$ \\
\hline
\end{tabular}

p1: $\mathrm{p}$ value between control and the NSAIDs group

$\mathrm{p} 2$ : $\mathrm{p}$ value between control and DMARDs group

p3: $p$ value between control and moderate disease activity group

$\mathrm{p} 4: \mathrm{p}$ value between control and high disease activity group

p5: $\mathrm{p}$ value between control and moderate and high disease activity group

p6: $p$ value between NSAIDs and DMARDs groups

p7: $\mathrm{p}$ value between moderate and high disease activity group

*: statistically significant at $\mathrm{p} \leq 0.05$

Table 4. Frequency of cells showing chromosomal aberrations in the control, RA patients on NSAIDs, DMARDs RA patients on MTX and MTX \& SSZ (out of 100 cells/ case).

\begin{tabular}{ccccccccc}
\hline & & Breaks & $\begin{array}{c}\text { Satellite } \\
\text { association }\end{array}$ & $\begin{array}{c}\text { Double } \\
\text { minutes }\end{array}$ & $\begin{array}{c}\text { Endo- } \\
\text { reduplication }\end{array}$ & Aneuploidy & $\begin{array}{c}\text { Other } \\
\text { aberrations }\end{array}$ & $\begin{array}{c}\text { Mitotic } \\
\text { index }\end{array}$ \\
\hline RA patients on & Range & $0-36$ & $15-48$ & $0-15$ & $0-16$ & $0-8$ & $0-2$ & $1.5-10.1$ \\
NSAIDs (10) & Mean \pm SD & $13.0 \pm 12.53$ & $28.7 \pm 12.78$ & $3.0 \pm 5.37$ & $5.6 \pm 5.83$ & $2.1 \pm 2.96$ & $0.4 \pm 0.699$ & $5.72 \pm 3.02$ \\
\hline RA patients on & Range & $0-43$ & $22-68$ & $0-20$ & $0-28$ & $0-13$ & $0-4$ & $2.4-10.1$ \\
MTX (12) & Mean \pm SD & $18.42 \pm 12.9$ & $41.33 \pm 15.92$ & $1.83 \pm 5.75$ & $11.0 \pm 7.59$ & $6.92 \pm 4.13$ & $0.42 \pm 1.21$ & $5.2 \pm 2.85$ \\
\hline RA patients on & Range & $4-25$ & $33-60$ & $0-4$ & $4-36$ & $0-11$ & $0-3$ & $3.4-13.5$ \\
MTX \& SZ (8) & Mean \pm SD & $11.5 \pm 7.23$ & $45.25 \pm 9.62$ & $1.13 \pm 1.64$ & $12.88 \pm 10.63$ & $6.88 \pm 4.11$ & $1.5 \pm 0.83$ & $7.38 \pm 3.02$ \\
\hline$P_{1}$ & & $>0.05$ & $<0.05^{*}$ & $>0.05$ & $<0.05^{*}$ & $<0.05^{*}$ & $>0.05$ & $>0.05$ \\
$\mathrm{P}_{2}$ & & $>0.05$ & $<0.05^{*}$ & $>0.05$ & $<0.05^{*}$ & $<0.05^{*}$ & $>0.05$ & $>0.05$ \\
$\mathrm{P}_{3}$ & & $>0.05$ & $>0.05$ & $>0.05$ & $>0.05$ & $>0.05$ & $>0.05$ & $>0.05$ \\
\hline
\end{tabular}

$\mathrm{p} 1: \mathrm{p}$ value between methotrexate group and NSAIDs group

p2: p value between methotrexate \& salzopyrin group and NSAIDs group

p3: p value between methotrexate group and methotrexate \& salzopyrin group

*: statistically significant at $\mathrm{p} \leq 0.05$ 
Tayel, et al.: Cytotoxicity and Genotoxicity in Patients with Rheumatoid Arthritis

Table 5. Frequency of cells showing chromosomal aberrations in the control and RA patients with different disease duration (out of 100 cells/ case).

\begin{tabular}{|c|c|c|c|c|c|c|c|}
\hline & & Breaks & $\begin{array}{c}\text { Satellite } \\
\text { association }\end{array}$ & $\begin{array}{l}\text { Double } \\
\text { minutes }\end{array}$ & $\begin{array}{c}\text { Endo- } \\
\text { reduplication }\end{array}$ & Aneuploidy & $\begin{array}{c}\text { Other } \\
\text { aberrations }\end{array}$ \\
\hline \multirow{2}{*}{ Control (10) } & Range & $0-3$ & $0-21.0$ & $0.0-0.0$ & $0.0-0.0$ & $0.0-0.0$ & $0.0-0.0$ \\
\hline & Mean \pm SD & $0.6 \pm 1.07$ & $6.2 \pm 6.49$ & $0.0 \pm 0.0$ & $0.0 \pm 0.0$ & $0.0 \pm 0.0$ & $0.0 \pm 0.0$ \\
\hline \multirow{2}{*}{$\begin{array}{l}\text { RA patients with disease } \\
\text { duration } 1-<5 \text { years }(13)\end{array}$} & Range & $3-43$ & $17-46$ & $0-2$ & $0-15$ & $0-10$ & $0-4$ \\
\hline & Mean \pm SD & $15.54 \pm 11.67$ & $32.38 \pm 8.97$ & $0.15 \pm 0.55$ & $6.77 \pm 5.07$ & $3.77 \pm 2.98$ & $0.46 \pm 0.98$ \\
\hline \multirow{2}{*}{$\begin{array}{l}\text { RA patients with disease } \\
\text { duration } 5-<10 \text { years }(9)\end{array}$} & Range & $0-36$ & $15-67$ & $0-15$ & $0-28$ & $0-8$ & $0-3$ \\
\hline & Mean \pm SD & $17.11 \pm 13.66$ & $39.78 \pm 8.53$ & $3.78 \pm 5.45$ & $11.78 \pm 9.44$ & $3.66 \pm 3.21$ & $0.78 \pm 0.384$ \\
\hline \multirow{2}{*}{$\begin{array}{l}\text { RA patients with disease } \\
\text { duration } 10-30 \text { years }(8)\end{array}$} & Range & $0-30$ & $25-68$ & $0-20$ & $0-36$ & $1-14$ & $0-3$ \\
\hline & Mean \pm SD & $10.88 \pm 9.11$ & $45.75 \pm 5.54$ & $3.13 \pm 6.92$ & $12.13 \pm 10.56$ & $7.70 \pm 4.47$ & $0.80 \pm 0.92$ \\
\hline $\mathrm{P} 1$ & & $0.001^{*}$ & $0.001^{*}$ & $0.001 *$ & $0.001^{*}$ & $0.001^{*}$ & $0.001^{*}$ \\
\hline $\mathrm{P}_{2}$ & & $0.001^{*}$ & $0.001^{*}$ & $0.001^{*}$ & $0.001 *$ & $0.001 *$ & $0.001^{*}$ \\
\hline $\mathrm{P}_{3}$ & & $0.001 *$ & $0.001 *$ & $0.001 *$ & $0.001 *$ & $0.001 *$ & $0.001 *$ \\
\hline
\end{tabular}

p1: $\mathrm{p}$ value between control and RA patients with disease duration between $1-<5$ years

p2: p value between control and RA patients with disease duration between $5-<10$ years

p3: p value between control and RA patients with disease duration between 10-30 years

*: statistically significant at $\mathrm{p} \leq 0.05$

Table 6. Frequency of cells showing chromosomal aberrations in the control, RA patients on DMARDs for different periods (out of 100 cells/ case).

\begin{tabular}{|c|c|c|c|c|c|c|c|}
\hline & & Breaks & $\begin{array}{c}\text { Satellite } \\
\text { association }\end{array}$ & $\begin{array}{l}\text { Double } \\
\text { minutes }\end{array}$ & $\begin{array}{c}\text { Endo- } \\
\text { reduplication }\end{array}$ & Aneuploidy & $\begin{array}{c}\text { Other } \\
\text { aberrations }\end{array}$ \\
\hline \multirow{2}{*}{ Control (10) } & Range & $0-3$ & $0-21.0$ & $0.0-0.0$ & $0.0-0.0$ & $0.0-0.0$ & $0.0-0.0$ \\
\hline & Mean \pm SD & $0.6 \pm 1.07$ & $6.2 \pm 6.49$ & $0.0 \pm 0.0$ & $0.0 \pm 0.0$ & $0.0 \pm 0.0$ & $0.0 \pm 0.0$ \\
\hline \multirow{2}{*}{$\begin{array}{l}\text { RA patients on DMARDs } \\
\text { for } 1-5 \text { years }(11)\end{array}$} & Range & $4-43$ & $22-60$ & $0-4$ & $0-20$ & $0-10$ & $0-4$ \\
\hline & Mean \pm SD & $17.64 \pm 11.63$ & $38.64 \pm 9.85$ & $0.55 \pm 1.29$ & $10.00 \pm 6.02$ & $4.67 \pm 3.50$ & $0.75 \pm 0.1 .22$ \\
\hline \multirow{2}{*}{$\begin{array}{l}\text { RA patients on DMARDs } \\
\text { for }>5 \text { years }(9)\end{array}$} & Range & $0-32$ & $25-68$ & $0-20$ & $0-36$ & $1-14$ & $0-3$ \\
\hline & Mean \pm SD & $13.22 \pm 11.05$ & $48.11 \pm 16.17$ & $2.78 \pm 6.55$ & $13.89 \pm 11.21$ & $7.45 \pm 4.32$ & $0.73 \pm 0.90$ \\
\hline $\mathrm{P}_{1}$ & & $0.001 *$ & $0.001^{*}$ & $0.001 *$ & $0.001^{*}$ & $0.001^{*}$ & $0.001^{*}$ \\
\hline $\mathrm{P}_{2}$ & & $0.001 *$ & $0.001 *$ & $0.001 *$ & $0.001 *$ & $0.001 *$ & $0.001 *$ \\
\hline $\mathrm{P}_{3}$ & & $0.021 *$ & $0.013 *$ & $0.036^{*}$ & $0.048 *$ & $0.036^{*}$ & 0.689 \\
\hline
\end{tabular}

p1: $\mathrm{p}$ value between control and RA patients on DMARDs for 1-5 years

$\mathrm{p} 2$ : $\mathrm{p}$ value between control and RA patients on DMARDs for $>5$ years

p3: $\mathrm{p}$ value between RA patients on DMARDs for 1-5 years and RA patients on DMARDs for $>5$ years

*: statistically significant at $\mathrm{p} \leq 0.05$

Table 7. Comparison between micronuclei (MN), nucleoplasmic bridges (NPB), and necrotic and/or apoptotic cells in rheumatoid arthritis patients and control.

\begin{tabular}{ccccc}
\hline & & BN cells with MN & BN cells with NPB & Necrotic and/or apoptotic cells \\
\hline \multirow{2}{*}{ Control (10) } & Total & 15 & 8 & 23 \\
& Range & $0-3$ & $0-3$ & $0-5$ \\
& Mean \pm SD & $1.5 \pm 1.2$ & $0.8 \pm 1.0$ & $2.3 \pm 1.8$ \\
\hline \multirow{2}{*}{ RA patients } & Total & 652 & 398 & 288 \\
$(30)$ & Range & $4-45$ & $3-40$ & $4-52$ \\
& Mean \pm SD & $21.73 \pm 12.38$ & $13.27 \pm 9.96$ & $29.93 \pm 12.10$ \\
\hline p value & & $<0.01 *$ & $<0.01 *$ & $<0.001 * *$ \\
\hline
\end{tabular}

Table 8. Comparison between the frequency distribution of the MN (by number) in RA patients and control.

\begin{tabular}{ccccc}
\hline & & $\mathbf{0 - 2}$ MN (Normal) & $\mathbf{3 - 5}$ MN (Moderate) & $>\mathbf{6}$ MN (High) \\
\hline \multirow{2}{*}{ Control (10) } & No & 8 & 2 & 0 \\
& $\%$ & 80 & 20 & 0 \\
\hline \multirow{2}{*}{ RA patients (30) } & No & 0 & 4 & 26 \\
\hline P-value & $\%$ & 0 & $<0.0001 *$ & 86.67 \\
\hline
\end{tabular}


Tayel, et al.: Cytotoxicity and Genotoxicity in Patients with Rheumatoid Arthritis

Table 9. Frequency of micronuclei, nucleoplasmic bridges (out of 1000 cells/ case), necrotic, and/or apoptotic cells (out of 500 cells/ case) in the study groups.

\begin{tabular}{|c|c|c|c|c|c|c|c|}
\hline & & $\begin{array}{c}\text { BN cells } \\
\text { with no } \\
\text { MN }\end{array}$ & $\begin{array}{c}\text { BN cells } \\
\text { with one } \\
\text { MN }\end{array}$ & $\begin{array}{c}\text { BN cells with } \\
\text { more than } \\
\text { one MN }\end{array}$ & $\begin{array}{c}\text { Total no of } \mathbf{B N} \\
\text { cells with } \\
\text { MNi }\end{array}$ & $\begin{array}{c}\text { BN cells } \\
\text { with } \\
\text { NPB } \\
\end{array}$ & $\begin{array}{c}\text { Necrotic and/or } \\
\text { apoptotic cells/ } \\
500 \text { cells }\end{array}$ \\
\hline \multirow{2}{*}{ Control (10) } & No. & 9985 & 9 & 6 & 15 & 8 & 23 \\
\hline & $\%$ & 99.85 & 0.09 & 0.06 & 0.15 & 0.08 & 0.46 \\
\hline \multirow{2}{*}{ RA patients on NSAIDs (10) } & No. & 9891 & 89 & 20 & 109 & 92 & 50 \\
\hline & $\%$ & 98.9 & 0.89 & 0.2 & 1.09 & 0.92 & 1.0 \\
\hline \multirow{2}{*}{ RA patients on DMARDs (20) } & No. & 19,457 & 453 & 90 & 543 & 306 & 238 \\
\hline & $\%$ & 97.29 & 2.27 & 0.45 & 2.72 & 1.53 & 2.38 \\
\hline \multirow{2}{*}{$\begin{array}{l}\text { RA patients with moderate } \\
\text { disease activity }(16)\end{array}$} & No. & 15,737 & 305 & 56 & 361 & 192 & 125 \\
\hline & $\%$ & 98.36 & 1.91 & 0.35 & 2.26 & 1.2 & 1.56 \\
\hline \multirow{2}{*}{$\begin{array}{l}\text { RA patients with high disease } \\
\text { activity (14) }\end{array}$} & No. & 13,704 & 237 & 54 & 291 & 206 & 163 \\
\hline & $\%$ & 97.89 & 1.69 & 0.39 & 2.08 & 1.47 & 2.33 \\
\hline \multirow{2}{*}{$\begin{array}{l}\text { RA patients with moderate and } \\
\text { high disease activity }(30)\end{array}$} & No. & 29,431 & 542 & 110 & 652 & 398 & 288 \\
\hline & $\%$ & 98.1 & 1.81 & 0.37 & 2.17 & 1.37 & 1.92 \\
\hline $\mathrm{P}_{1}$ & & $>0.05$ & $<0.05^{*}$ & $>0.05$ & $<0.05^{*}$ & $<0.05 *$ & $<0.05^{*}$ \\
\hline $\mathrm{P}_{2}$ & & $>0.05$ & $<0.05^{*}$ & $>0.05$ & $<0.05^{*}$ & $<0.05^{*}$ & $<0.05^{*}$ \\
\hline $\mathrm{P}_{3}$ & & $>0.05$ & $<0.05^{*}$ & $>0.05$ & $<0.05^{*}$ & $<0.05^{*}$ & $<0.05^{*}$ \\
\hline $\mathrm{P}_{4}$ & & $>0.05$ & $<0.05^{*}$ & $>0.05$ & $<0.05^{*}$ & $<0.05^{*}$ & $<0.05^{*}$ \\
\hline $\mathrm{P}_{5}$ & & $>0.05$ & $<0.05^{*}$ & $>0.05$ & $<0.05^{*}$ & $<0.05 *$ & $<0.05^{*}$ \\
\hline $\mathrm{p}_{6}$ & & $>0.05$ & $<0.05^{*}$ & $>0.05$ & $>0.05$ & $>0.05$ & $>0.05$ \\
\hline P7 & & $>0.05$ & $>0.05$ & $>0.05$ & $>0.05$ & $>0.05$ & $>0.05$ \\
\hline
\end{tabular}

p1: $\mathrm{p}$ value between control and the NSAIDs group

$\mathrm{p} 2$ : $\mathrm{p}$ value between control and DMARDs group

p3: $\mathrm{p}$ value between control and moderate disease activity group

p4: $\mathrm{p}$ value between control and high disease activity group

$\mathrm{p} 5$ : $\mathrm{p}$ value between control and moderate and high disease activity group

p6: $p$ value between NSAIDs and DMARDs groups

$\mathrm{p} 7: \mathrm{p}$ value between moderate and high disease activity group

p: $\mathrm{p}$ value for chi-square test $\quad *$ : statistically significant at $\mathrm{p} \leq 0.05$

Table 10. Frequency of micronuclei, nucleoplasmic bridges (out of 1000 cells/ case), necrotic, and/or apoptotic cells (out of 500 cells/ case) in the control, RA patients on NSAIDs, DMARDs RA patients on MTX and MTX \& SSZ the study groups.

\begin{tabular}{|c|c|c|c|c|c|c|c|}
\hline & & $\begin{array}{l}\text { BN cells with } \\
\text { no } \mathrm{MNi}\end{array}$ & $\begin{array}{l}\text { BN cells with } \\
\text { one } M N\end{array}$ & $\begin{array}{c}\text { BN cells with } \\
\text { more than one } \\
\text { MN }\end{array}$ & $\begin{array}{l}\text { Total no of } \mathrm{BN} \\
\text { cells with } \mathrm{MNi}\end{array}$ & $\begin{array}{c}\text { BN cells with } \\
\text { NPB }\end{array}$ & $\begin{array}{c}\text { Necrotic } \\
\text { and/or } \\
\text { apoptotic cells/ } \\
500 \text { cells }\end{array}$ \\
\hline \multirow{2}{*}{$\begin{array}{c}\text { RA patients on NSAIDs } \\
(10)\end{array}$} & No. & 9891 & 89 & 20 & 109 & 92 & 50 \\
\hline & $\%$ & 98.9 & 0.89 & 0.2 & 1.09 & 0.92 & 1.0 \\
\hline \multirow{2}{*}{$\begin{array}{c}\text { RA patients on MTX } \\
\text { (12) }\end{array}$} & No. & 11673 & 285 & 42 & 327 & 180 & 142 \\
\hline & $\%$ & 97.28 & 2.38 & 0.35 & 2.73 & 1.5 & 2.37 \\
\hline \multirow{2}{*}{$\begin{array}{c}\text { RA patients on MTX \& } \\
\text { SZ (8) }\end{array}$} & No. & 7784 & 168 & 48 & 216 & 126 & 96 \\
\hline & $\%$ & 97.3 & 2.1 & 0.6 & 2.7 & 1.58 & 2.4 \\
\hline $\mathrm{P} 1$ & & $>0.05$ & $<0.05^{*}$ & $>0.05$ & $>0.05$ & $<0.05^{*}$ & $<0.05^{*}$ \\
\hline $\mathrm{P}_{2}$ & & $>0.05$ & $<0.05^{*}$ & $>0.05$ & $>0.05$ & $<0.05^{*}$ & $<0.05^{*}$ \\
\hline P3 & & $>0.05$ & $>0.05$ & $>0.05$ & $>0.05$ & $>0.05$ & $>0.05$ \\
\hline
\end{tabular}

P1: $\mathrm{p}$ value between MTX group and NSAIDs group

P2: $\mathrm{p}$ value between MTX \& SSZ group and NSAIDs group

P3: p value between MTX group and MTX \& SZ group

$\mathrm{p}$ : $\mathrm{p}$ value for chi-square test* ${ }^{*} \quad$ Statistically significant at $\mathrm{p} \leq 0.05$ 

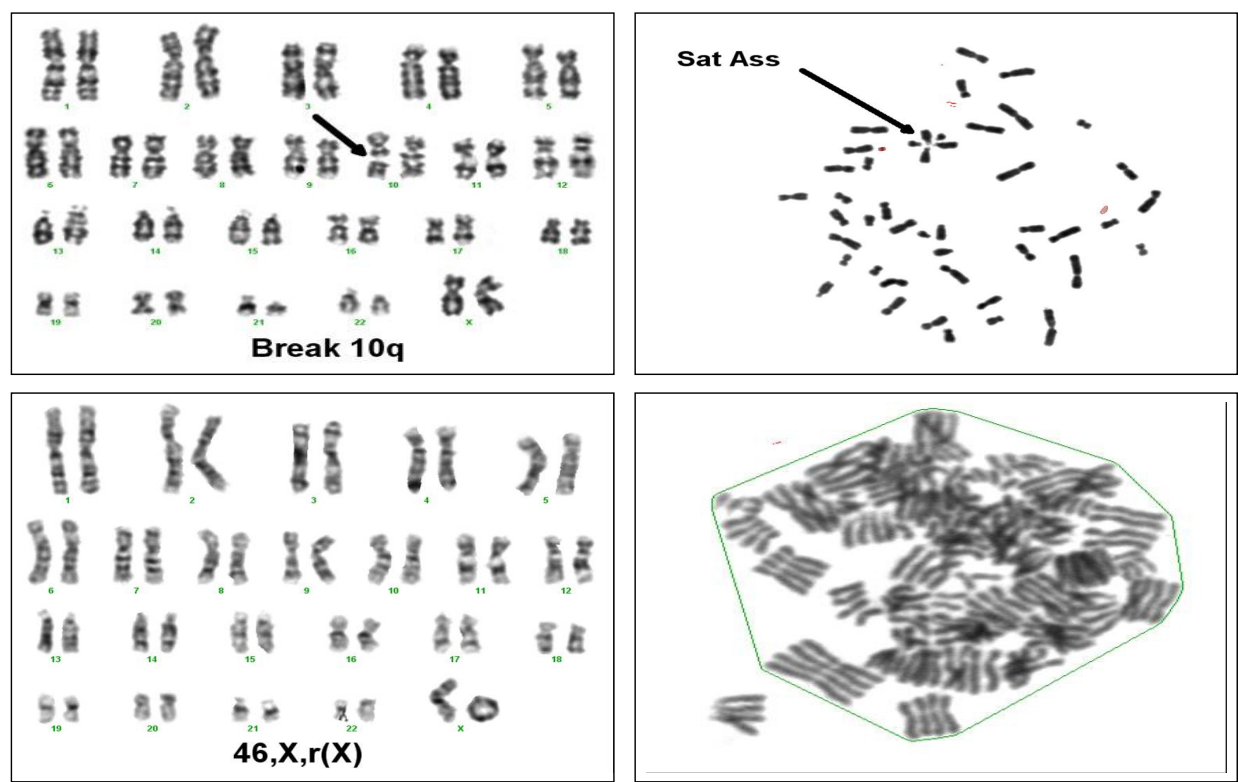

Figure 1. Photograph of a GTG karyotype of a RA patient showing break in the long arm of chromosome 10 (10q, Upper left), Photograph of a metaphase spread (Giemsa stained) of a RA patient showing satellite association (Sat Ass) between 3 chromosomes of the D group and 2 of the $\mathrm{G}$ group (Upper right), Photograph of a karyotype of RA patient (GTG- banding) showing ring X- chromosome (lower left) and photograph of metaphase spread of a RA patient stained by Giemsa showing chromosome endoreduplication (Lower right).
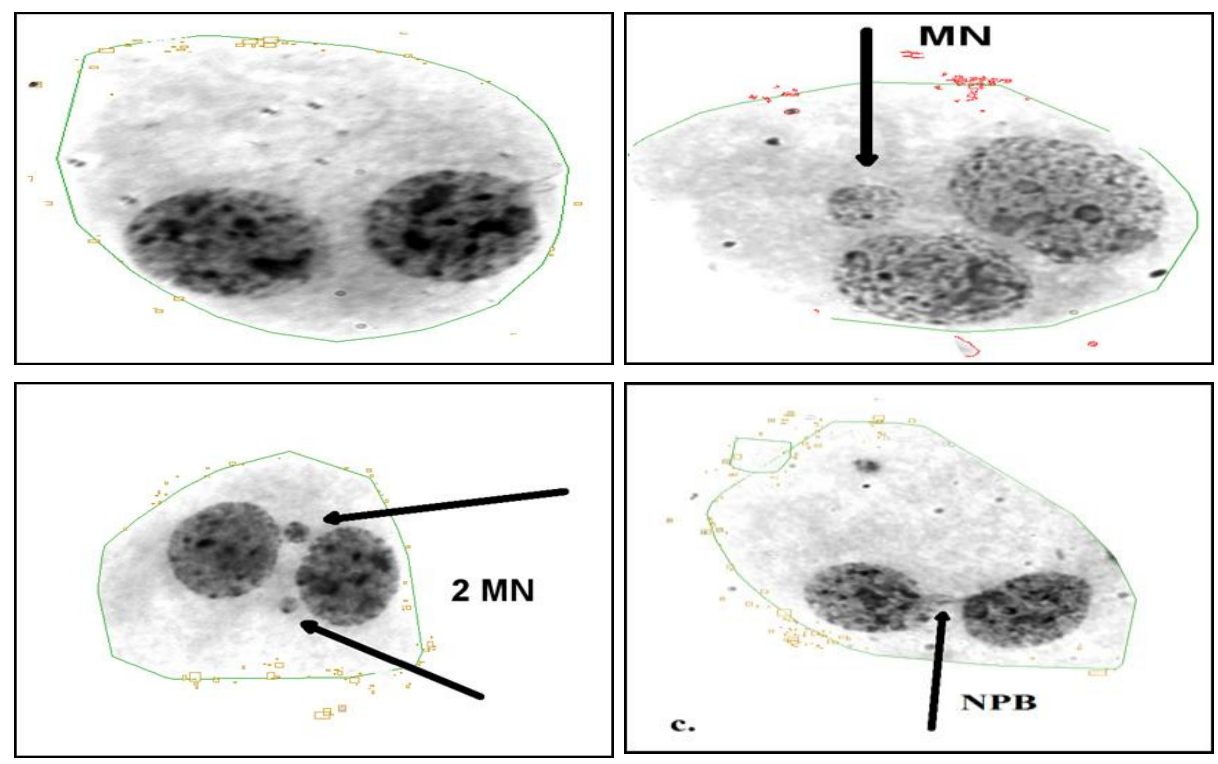

Figure 2. Photograph of a normal binucleated (BN) cell (Upper left), Photograph of a binucleated cell with one micronucleus (MN) observed in RA patient (Upper right), Photograph showing a binucleated cell with more than one micronucleus (MN) seen in RA patient (Lower left), and photograph of a binucleated cell with nucleoplasmic bridge (NPB) observed in RA patient (Lower right). 


\section{DIscussion}

The "Bermuda triangle" of genetics, environment and autoimmunity is involved in the pathogenesis of RA. ${ }^{4}$ The disease has a heterogeneous character. Although the pathogenesis of RA is incompletely understood, genetic factors play a vital role in susceptibility to RA as the heritability of RA is between 50 and $60 \%{ }^{21}$.

Bhatia et al. ${ }^{22}$ have compared the prevalence of rheumatoid factor, which is often present before the development of RA in individuals on oral contraceptive pills (OCPs) with the hypothesis that OCPs would be protective, and therefore individuals on OCPs would have a lower prevalence of rheumatoid factor than those who are not. This result suggests a protective effect of OCPs with regards to the development of rheumatoid factor, but not necessarily for RA. Ramos-Remus et al. ${ }^{15}$ have found no significant association between the $\mathrm{MN}$ number and the contraceptive use in the RA patients of their study. In the present study the opposite was observed were 18 cases $(60 \%)$ with RA were on or have taken OCPs (for more than 5 years) and were rheumatoid factor positive so denoting that OCPs neither protect against the development of rheumatoid factor nor RA.

Amelioration of RA has been observed during pregnancy with exacerbation in the postpartum period. Therefore, investigators sought to assess whether risk of disease is modified by breastfeeding. A recent study found that long-term breast feeding of greater than or equal to 13 months was associated with a significant reduction of the risk of $\mathrm{RA}^{23}$. These findings corroborate with a previous study which found that breast feeding more than 12 months was inversely related to the development of RA with a lower risk with longer duration of breast feeding ${ }^{24}$. In the present study 21 RA patients $(75 \%)$ out of 28 who had children have confirmed less severity of the RA disease during pregnancy while the answers were inconclusive in the other 7 patients. Breast feeding for more than a year was also associated with less disease activity in 17 cases $(81 \%)$ out of the 21 cases who had children and breast fed their babies. These findings are in agreement with the previous data about the protective effect of pregnancy and breast feeding.

There is an inverse association between socioeconomic status measured by education and occupational class and risk of $\mathrm{RA}^{25}$. In the present study all cases were of low socioeconomic class, rheumatoid factor positive and 28 of them $(93.3 \%)$ were without university degree so confirming the above observation about the socioeconomic status.

Increased chromosomal breakage is a common feature of some disorders such as lupus erythematosus, dermatomyositis, periarteritis nodosa and rheumatoid arthritis ${ }^{26}$. Kinne et al. 2001. ${ }^{27}$ have illustrated that chromosomal aberrations were observed in synovial tissue and synovial fluid fibroblasts (SFB) of patients with RA, OA, and other inflammatory joint diseases while no aberrations were observed in the peripheral blood of the same patients. The study performed by Vincent et al. ${ }^{28}$ confirmed the significant elevation of the rate of chromosomal abnormalities and the presence of a breaking capacity of the serum in a series of 78 rheumatoid polyarthritis compared with a control group. These lesions were observed from the onset of the disease but were not specific of this disease. They concluded that chromosomal abnormalities do not seem to be randomly distributed on the chromosomes. They are not correlated with clinical or biological parameters in a statistically significant fashion. In the present study a significant increase in chromosomal aberrations in the form of breaks, Sat Ass, dmin, endoreduplication, aneuploidy and other aberrations was observed in the 30 RA patients compared to control so suggesting that cytotoxicity is a manifestation of RA disease itself. This significant increase in chromosomal aberrations in RA patients was not correlated to the degree of disease activity (where no significance difference was noted between patients with moderate and high disease activity). There was a highly significant difference $(p \leq 0.001)$ in all the studied markers of cytotoxicity between the control and RA patients irrespective of the disease duration (RA patients with disease durations between $1-<5$ years, $5-<10$ years, and 10-30 years) so documenting that cytotoxicity occurs with the onset of the disease and is a manifestation of it. These findings match the findings observed by Emerit $^{26}$ and Vincent et al. ${ }^{28}$ but in contradiction with that of Kinne et al. ${ }^{27}$. The other chromosomal aberrations encountered in the present study (as loss or gain of some chromosomes) seem to be randomly distributed except in the case of $\mathrm{X}$ chromosome anomalies as it occurred in 6 patients (30\%) receiving DMARDs (out of 20 patients) in the form of monosomy $\mathrm{X}, \mathrm{Xq}-$, and ring $\mathrm{X}$.

Among the various DNA lesions induced by MTX, chromosome breaks are considered the most important because of their potential to cause cell death, mutagenesis, and carcinogenesis ${ }^{29}$. Shahin et al. $^{30}$ have observed that MTX in a dose of 0.5 $\mathrm{mg} / \mathrm{kg} /$ day produced a significant genetic injury as proved by the increased incidence of $\mathrm{CAs}$ and $\mathrm{MNi}$ formation in Wistar albino rats. They performed a study on 20 patients with RA $(5$ males and 15 females) treated with $10 \mathrm{mg}$ weekly dose of MTX, i.m., for one month, followed by the administration of the same dose of MTX in addition to $10 \mathrm{mg}$ of folinic 
acid as a single dose 4 hours after MTX administration, i.m., every week for another 4 weeks. Chromosomal studies as well as a micronucleus test were then evaluated for each patient. Their results showed that MTX produced a significant genetic injury as proved by the increased incidence of CAs and MNi. Inversely, folinic acid produced a satisfactory improvement of MTX-induced genetic damage in RA patients. They concluded that folinic acid has a protective effect against MTX genotoxicity in patients with RA. Similar results were reported by Theiss et al. ${ }^{31}$ and Mac Donald et al. ${ }^{32}$, whose studies showed an increase in trimetrexate (TMX) - a drug structurally similar to MTX - genotoxicity, as evidenced by increased CAs in TMX-treated rats.

In the present study there was a significant difference in CAs between RA patients on DMARDs and control. In the same respect, there was a significant difference between RA patients on MTX and those on NSAIDs as regards Sat Ass, endoreduplication, and aneuploidy which indicates the cytotoxicity of MTX. The other CAs studied in MTX patients that showed no statistically significant difference between MTX and NSAIDs RA patients i.e. breaks, dmin, and other aberrations might be masked by the protective effect of folic acid used concurrently with MTX.

Sat Ass is known to occur in meiosis as well as in mitosis where the respective chromosomes (meiosis) or chromatids (mitosis) may fail to disjoin so leading to chromosomal aneuploidy. ${ }^{33}$. Lezhava et al. ${ }^{34}$ have mentioned that the associations of human acrocentric chromosomes account for elevated incidence of chromosome rearrangements and consequently, can cause chromosomal disorders. The normal range of Sat Ass established by examining 1161 metaphase plates of 22 controls ranged from 2$27 \%$ with a mean of $13.3 \%{ }^{33}$. In the present study, the mean of Sat Ass in the control group was 6.2 \pm 6.49 , in RA patients was $38.16 \pm 14.77$. Rheumatoid arthritis patients of the present study had a significantly high frequency of Sat Ass which in turn can explain the high frequency of aneuploidy observed in these cases. This high frequency of Sat Ass can also explain the high incidence of abortions in RA patients of the present study (11 cases; $39.3 \%, 8$ of them i.e. $72.7 \%$ were using DMARDs) which might be due to chromosome non-disjunction occurring in meiosis. To the best of our knowledge no study on the Sat Ass in patients with RA was found in the literature.

Cronstein $^{35}$ has mentioned that a great percentage of patients continued to take MTX for their RA for longer than any other second-line agent as it is more effective and well tolerated although toxicities were encountered. $\mathrm{He}$ also added that concomitant administration of either folinic acid or folic acid to patients with RA taking MTX demonstrated no difference in therapeutic efficacy of the MTX and prevention of methotrexate-mediated toxicity. However, the most recent guidelines issued by the American College of Rheumatology for the therapy of RA include the suggestion that folic acid or folinic acid may be useful in the prevention of complications of MTX therapy ${ }^{36}$. Ferraccioli et al. ${ }^{37}$ - after analysis of the response and toxicity of 126 patients with early active RA treated with MTX (with a dose of 10-20 $\mathrm{mg} /$ week), and sulphasalazin (dose of $1-3 \mathrm{~g} /$ day) have stated that in long term treatment programmes the safety issue must receive considerable attention.

In the current study, dmin, endoreduplication, aneuploidy, and other chromosomal aberrations showed a statistically significant increase in RA patients on MTX than control. The results also showed a high significant difference in all the cytogenetic markers examined between the control and RA patients on DMARDs irrespective of the time of use (for a period of 1-5 years, and > 5 years where $\mathrm{p} 1$ and $\mathrm{p} 2$ where $\leq 0.001$ ) so denoting the cytotoxicity of DMARDs used in the present study. RA patients who where on DMARDs for a longer duration ( $>5$ years) showed a statistically significant increase in all the cytogenetic markers (except other chromosomal aberrations; $\mathrm{p} 3$ was $\leq 0.05$ ) so illustrating that the cytotoxicity of DMARDs increases with the long duration of use ( $>5$ years). These observations are in agreement with the above mentioned observations cited by Cronstein ${ }^{35}$ and Ferraccioli et al. ${ }^{37}$ about the toxicity of MTX.

Compared with the general population, patients with RA have a higher risk of developing certain malignancies ${ }^{38}$. A number of studies have found an increased risk of malignancy in RA patients treated with disease-modifying antirheumatic drugs (DMARDs). Methotrexate may be associated with hemopoietic malignancies, although the literature consists predominantly of case reports ${ }^{39}$. In a casecontrol study, Williams et al. ${ }^{39}$ found that RA patients with leukemia or lymphoma showed a trend toward increased prior methotrexate or azathioprine use compared with matched RA controls without leukemia or lymphoma. The high frequency of dmin, and endoreduplication observed in the DMARDs and MTX groups of the present study can lead to increased risk of malignancy in RA patients so explaining the observations made by Woodrick and Ruderman ${ }^{38}$ and Williams et al. ${ }^{39}$.

Studies in vitro and in experimental animals have reported that MTX may induce MN in a dose dependent manner ${ }^{40,41}$, and that folinic acid supplementation reduces the cytogenetic damage induced by $\mathrm{MTX}^{42}$. Yet to the best of our knowledge, $\mathrm{MN}$ induction has not been studied from peripheral blood in RA patients using MTX. 
Iatropoulos et al. ${ }^{43}$ have cited that SASP, which has been in clinical use for over 50 years, was reported by the National Toxicology Program to increase micronuclei $(\mathrm{MN})$ in cultured human lymphocytes. They found that treatment with folate has reduced the frequency of MN. They added that folate deficiency associated with SASP administration is probably responsible for aneuploidy in lymphocytes. Accordingly, SASP is judged to pose no carcinogenic risk to humans.

On the contrary, The National Toxicology Program $^{44}$ results of micronucleus assays performed on male or female mice exposed to SASP indicated that it is capable of inducing chromosomal damage, possibly in the form of aneuploidy, in mouse bone marrow cells after multiple administrations.

In the present study there was a significant difference between RA patients on MTX \& SSZ and those on NSAIDs as regards Sat Ass, endoreduplication and aneuploidy only while the difference in the other chromosomal anomalies as breaks, dmin, and other aberrations was not statistically significant. In the same time no significant difference was observed between the MTX group and the MTX \& SZ one.

The same results have been achieved as regards the $\mathrm{MN}$ test where significant difference was seen between RA patients on MTX \& SSZ when compared to control as regards the frequency of $\mathrm{BN}$ cells with $\mathrm{NPB}$, one (not more) $\mathrm{MNi}$, and the necrotic and or apoptotic cells while no significant difference was observed between the MTX group and the MTX \& SSZ one. These findings indicate whether that SSZ is not toxic (as it did not augment the MTX toxicity) or that its toxicity has been masked by the use of folic acid. Further studies are needed on RA patients using SSZ alone (not in combination with MTX) with and without folic acid use to delineate the cyto- and genotoxicity of SSZ.

In a series of studies ${ }^{45-47}$ on folic acid deficiency in long-term primary human lymphocyte cultures, the interrelationship between $\mathrm{MNi}$, NPBs and NBUDs (biomarkers of chromosome damage) and the impact of folic acid deficiency, has been quantified. Folic acid concentration correlated significantly and negatively with all these markers of chromosome damage. Karaman et al. ${ }^{48}$ have found that the MN frequencies were significantly higher in RA patients both in the active and the inactive period- than in the controls. Their results suggested that the higher $\mathrm{MN}$ frequency (the DNA damage) in RA can be explained by increased oxidative stress (as evidenced by increased production of ROS) so leading to genetic instability. They suggested that increased DNA damage may play an important role in the pathogenesis of RA.
Ramos-Remus ${ }^{15}$ have performed MN assays on oral mucosal sweeps of 50 MTX treated RA patients, 30 RA patients not receiving MTX and 39 healthy controls. Then they randomly placed the MTX treated RA patients in a cross-over design to receive folic acid supplementation, and MN assays were repeated after 6 weeks. They found that the number of $\mathrm{MN}$ were significantly higher in RA patients as compared with controls. However, no difference in the MN number was observed between users and non-users of MTX. $\mathrm{MN}$ in RA patients were more frequently in the moderate to high range compared with controls, whether or not they were users of MTX. They concluded that genotoxicity, as assessed by the MN assay, is increased in RA patients. They also added that these results suggest that genotoxicity is associated with RA itself and not with MTX use. Folic acid supplementation had no effect on the number of MN.

In the present study two different groups of RA patients were assembled. The first group consisted of RA patients taking DMARDs (MTX or MTX \& SSZ) for different durations. The second group consisted of RA patients who had never used MTX or SSZ. MN test was performed in all of these patients and the results were compared with healthy controls. These groups allowed an assessment of whether RA patients who were or were not using MTX have more MN than healthy controls.

In the present study there was a statistically significant increase $(\mathrm{p}<0.01)$ in the mean $\mathrm{MNi}$ $(21.73 \pm 12.38)$ and the mean NPB $(13.27 \pm 9.96$, $\mathrm{p}<0.01)$ in RA patients compared with controls (means of $1.5 \pm 1.2$ and $0.8 \pm 1$ for $\mathrm{MNi}$ and $\mathrm{NPB}$ respectively), regardless of their MTX use. This suggests that RA itself rather than MTX induces $\mathrm{MNi}$, which is in accord with the suggestion that the increased risk of leukemia may be due to RA itself ${ }^{39}$.

The number of MN detected in any person is classified as normal when lies between 0-2, moderate (3-5), or high if $\geq 6$. The number of $\mathrm{MNi}$ in the $30 \mathrm{RA}$ patients in the current study was high in $86.67 \%$ ( $>6$ $\mathrm{MNi})$, and moderate in $13.33 \%(3-5 \mathrm{MNi})$ as compared to the control group where $80 \%$ had the normal number of $\mathrm{MNi}(0-2)$. This difference in the $\mathrm{MNi}$ number was highly significant $(<0.0001)$ so documenting the genotoxicity of RA. This finding is also in agreement with that of Ramos-Remus et al. ${ }^{15}$ and Shahin et al. ${ }^{30}$. There was a significant difference in $\mathrm{BN}$ cells with one $\mathrm{MNi}$ (not more than one), NPB and necrotic and/or apoptotic cells between the MTX group and the NSAIDs one so documenting the genotoxicity of MTX and the partial protective effect of folic acid. Theses findings are also in agreement with the results of Shahin et al. ${ }^{30}$.

Shahin et al. ${ }^{30}$ stated that the MTX-induced micronuclei formation might be explained by the 
intracellular accumulation of the drug resulting in a continuous inhibition of deoxyribonucleotide triphosphate (dNTPs) synthesis, subsequently causing DNA lesions due to the inhibition of DNA repair. However, because insufficient dNTPs remain, DNA lesions induced by MTX genotoxicity present themselves as micronuclei. Jia et al. ${ }^{49}$ stated that MTX can cause the formation of acentric chromosome fragments and the malsegregation of whole chromosomes. Acentric chromosome fragments and whole chromosomes that are unable to interact with the spindle lag behind at anaphase, and as a result, they are not included in the main daughter nuclei. A lagging chromosome fragment or whole chromosome forms in a small separate nucleus; hence, the term micronucleus.

The genotoxicity estimation for the MTX cases of the present study, as assessed by MN test, was consistent with that determined by karyotyping which suggests that both the MN assay and chromosome aberration analysis are capable of providing reliable cytogenetic damage assessment.

In conclusion, $\mathrm{CAs}, \mathrm{MNi}$, and $\mathrm{NPB}$ were increased RA patients so suggesting that cytotoxicity and genotoxicity are manifestations of RA disease itself. These CAs, MNi, and NPB were not correlated to the degree of disease activity, and seemed to be randomly distributed except in the case of $\mathrm{X}$ chromosome anomalies. MTX is still cyto-and genotoxic to RA patients despite the associated use of folic acid. This toxicity might be due to the high dose and its long time of use in the present study. OCPs neither protect against the development of rheumatoid factor nor RA. Pregnancy and breast feeding have a protective effect against RA. There is an inverse association between socioeconomic status measured by education and occupational class and risk of RA.

1. Liao KP, Lars A, Elizabeth WK. Environmental influences on risk for rheumatoid arthritis. Curr Opin Rheumatol. 2009; 21(3): 279-283.

2. Collignon O. Methodological issues in the design of a rheumatoid arthritis activity score and its cutoffs. Clinic Epidemiol. 2014; (4:6):221-6.

3. Ivanio AP, Licia MH, Boris AC, Claiton VB, Lucila SR, et al. Brazilian society of rheumatology consensus on the management of comorbidities in patients with rheumatoid arthritis. Rev Bras Reumatol. 2012; 52(4):474-95.

4. Júlia K, Timea B, Judit L, Tibor TG, Katalin M, Zoltán S. Genetics of Rheumatoid Arthritis - A Comprehensive Review. Clin Rev Allergy Immunol. 2013; 45(2): 170-9.
5. Salavoura K, Dracou C, Kolialexi A, Drakonaki S, Sofocleous C, Mavrou A. Juvenile idiopathic arthritistype disease associated with chromosomal aberrations. Clin Exp Rheumatol. 2008;26(2):347-50.

6. Czakó M, Riegel M, Morava E, Schinzel A, Kosztolányi G. Patient with rheumatoid arthritis and MCA/MR syndrome due to unbalanced der (18) transmission of a paternal translocation $\mathrm{t}(18 ; 20)(\mathrm{p} 11.1 ; \mathrm{p} 11.1)$. Am J Med Genet. 2002;108(3):226-8.

7. Maxwell JR, Marinou I, Kuet KP, Orozco G, Moore DJ, Barton A, Worthington J, Wilson AG. Rheumatoid arthritis-associated polymorphisms at $6 q 23$ are associated with radiological damage in autoantibody-positive RA. J Rheumatol. 2012; 39(9): 1781-5.

8. Orozco G, Viatte S, Bowes J, Martin P, Wilson AG, et al. UK Rheumatoid Arthritis Genetics Consortium; Wellcome Trust Case Control Consortium; Biologics in Rheumatoid Arthritis Genetics and Genomics Study Syndicate Consortium, Barton A, Worthington J, Eyre $\mathrm{S}$. Novel rheumatoid arthritis susceptibility locus at 22q12 identified in an extended UK genome-wide association study. Arthritis Rheumatol 2014; 66(1): 24-30.

9. Gabay C, Hasler P, Kyburz D, So A, Villiger P, von Kempis J, Walker U. Biological agents in monotherapy for the treatment of rheumatoid arthritis. Swiss Med Wkly. 2014 (10);144:w13950.

10. Saeed SA, Gilani AH, Rasheed H, Bhatti FN, Atiq $\mathrm{M}$, et al. Plasma from rheumatoid patients taking low dose methotrexate enhances platelet aggregation. Res Commun Mol Pathol Pharmacol. 2002;111(1-4):69-76.

11. Suarez Almazor ME, Belseck E, Shea B, Tugwell P, Wells GA. Sulfasalazine for treating rheumatoid arthritis. Cochrane Database of Systematic Reviews 2000;2 CD000958.

12. Linares V, Alonso V, Domingo JL. Oxidative stress as a mechanism underlying sulfasalazine-induced toxicity. Expert Opin Drug Saf. 2011;10(2):253-63.

13. Bolognesi C, Landini E, Roggieri P, Fabbri R. Genotoxicity biomarkers in the assessment of heavy metal effects in mussels: experimental studies. Environ Mol Mutagen 1999; 33(4): 287-92.

14. Fenech M. Cytokinesis-block micronucleus cytome assay. Nature Protocols. 2007;2(5):1084-1104.

15. Ramos-Remus C, Dorazco-Barragan G, AcevesAvila FJ, Alcaraz-Lopez F, Fuentes-Ramirez F et al. Genotoxicity assessment using micronuclei assay in rheumatoid arthritis patients. Clin Exp Rheumatol 2002; 20(2):208-12.

16. David TF, Josef SS, George W, Bin Z, Lilian HD. American College of Rheumatology/European League against Rheumatism Preliminary. Definition of Remission in Rheumatoid Arthritis for Clinical Trials. Arthritis Rheum. 2011; 63(3):573-86. 
17. Sharma AK, Sharma A. Chromosome analysis following short and long term culture in animals including man. In: Chromosome techniques. Butterworth and Co. Ltd. London, Boston, Sydney, Durban, Toronto 1980; 361-2.

18. Shaffer LG, Mc Gowan-Jordan J, Schmid M. An international system for human cytogenetic nomenclature. ISCN 2013; 978(3-318):2253-7.

19. Fenech M, Chang WP, Kirsch-Volders M, Holland N, Bonassi S, Zeiger E; Human Micronucleus project. HUMN project: detailed description of the scoring criteria for the cytokinesis-block micronucleus assay using isolated human lymphocyte cultures. Muta Res 2003; 534(1-2):65-75.

20. Altman GA (1991) (ed) Practical Statistics for Medical Research, 1st ed., Chapman \& Hall, London.

21. Vasanth KM, Nalini G, Rajasekhar G. Association of susceptible genetic markers and autoantibodies in rheumatoid arthritis. J Genetics. 2014; 93(2):597-605

22. Bhatia SS, Majka DS, Kittelson JM, et al. Rheumatoid factor seropositivity is inversely associated with oral contraceptive use in women without rheumatoid arthritis. Ann Rheum Dis 2007;66:267-269.

23. Pikwer M, Bergström U, Nilsson JA, Jacobsson L, Berglund G, Turesson C. Breast feeding, but not use of oral contraceptives, is associated with a reduced risk of rheumatoid arthritis. Ann Rheum Dis. 2009;68(4):526-30.

24. Karlson EW, Mandl LA, Hankinson SE, Grodstein F. Do breast-feeding and other reproductive factors influence future risk of rheumatoid arthritis? Results from the Nurses' Health Study. Arthritis Rheum 2004;50:3458-67.

25. Bengtsson C, Nordmark B, Klareskog L, et al. Socioeconomic status and the risk of developing rheumatoid arthritis: results from the Swedish EIRA study. Ann Rheum Dis 2005;64:1588-94.

26. Emerit I. Chromosomal breakage in systemic sclerosis and related disorders. Dermatologica. 1976;153(3):145-56.

27. Kinne RW, Liehr $\mathrm{T}$, Beensen $\mathrm{V}$, Kunisch E, Zimmermann $\mathrm{T}$, et al. Mosaic chromosomal aberrations in synovial fibroblasts of patients with rheumatoid arthritis, osteoarthritis, and other inflammatory joint diseases. Arthritis Res. 2001;3(5):319-30.

28. Vincent G, Croquette MF, Houvenagel E, Leloire O. Chromosome anomalies in rheumatoid polyarthritis. Breakage level and study of the breaking factor. Rev Rhum Mal Osteoartic. 1986 Nov;53(11):625-30.

29. Osipov, A.N.; Buleeva, G.; Arkhangelskaya, E.; Klokov, D. In vivo $\gamma$-irradiation low dose threshold for suppression of DNA double strand breaks below the spontaneous level in mouse blood and spleen cells. Mutat Res 2013;(30)756(1-2):141-5.
30. Shahin AA, Ismail MM, Saleh AM, Moustafa HA, Aboul-Ella AA, Gabr HM. Protective effect of folinic acid on low-dose methotrexate genotoxicity. Z Rheumatol. 2001;60(2):63-8.

31. Theiss JC, Krishma G, Kropko M et al. Effect of leucovorin on the in vitro cytotoxic and clastogenic activity of trimetrexate. Proc Am Assoc. Cancer Res 1989;30:472-76.

32. Mac Donald JR, Courtney DG, Pegg DG. Leucovorin protection against repeated daily dose toxicity of trimetrexate in rats. Fund Appl Toxicol 1993;21:244-52.

33. Zellweger H, Abbo G, Cuany R. Satellite association and translocation mongolism. J Med Genet 1966;3:186-89.

34. Lezhava T, Tsigroshvili Z, Dvalishvili N, Jokhadze T. Mathematical model for satellite associations of human acrocentric chromosomes. Georgian Med News. 2008;164: 90-9.

35. Cronstein BN. Low-dose methotrexate: a mainstay in the treatment of rheumatoid arthritis. Pharmacol Rev. 2005;57(2):163-72.

36. American College of Rheumatology Subcommittee on Rheumatoid Arthritis Guidelines. Guidelines for the management of rheumatoid arthritis. Arthritis Rheum 2002;46:328-46.

37. Ferraccioli GF, Gremese E, Tomietto P, Favret G, Damato R, Di Poi E. Analysis of improvements, full responses, remission and toxicity in rheumatoid patients treated with step-up combination therapy (methotrexate, cyclosporine A, sulphasalazine) or monotherapy for three years. Rheumatology 2002; 41:892-98.

38. Woodrick RS, Ruderman EM. Safety of biologic therapy in rheumatoid arthritis. Nat Rev Rheumatol 2011;7:639-52.

39. Williams CA, Bloch DA, Sibley J, et al. Lymphoma and leukemia in rheumato id arthritis: are they associated with azathioprine, cyclophosphamide, or methotrexate ? A matched case-control study in the Arthritis, Rheumatism and Aging Medical Information System (ARAMIS) population. J Clin Rheumatol 1996;2:64-72.

40. Kasahara Y, Nakai Y, Miura D, Yagi K, Hirabayashi K, Makita T. Mechanism of induction of micronuclei and chromosome aberrations in mouse bone marrow by multiple treatments of methotrexate. Mutat Res 1992;280:117-28.

41. Kasahara Y, Wakata A, Nakai Y. The micronucleus test using peripheral blood reticulocytes from methotrexate-treated mice. Mutat Res 1992;278:145-51.

42. Keshava C, Keshava N, Whong W et al. Inhibition of methotrexate induced chromosomal damage by folinic acid in V79 cells. Mutation Res 1998; 397: 221-28.

43. Iatropoulos MJ, Williams GM, Abdo KM, Kari FW, Hart RW. Mechanistic studies on genotoxicity and carcinogenicity of salicylazosulfapyridine an anti-inflammatory medicine. Exp Toxicol Pathol 1997;49(1-2):15-28. 
44. NTP Toxicology and Carcinogenesis Studies of Salicylazosulfapyridine (CAS No. 599-79-1) in F344/N Rats and B6C3F1 Mice (Gavage Studies). National Toxicology Program. Natl Toxicol Program Tech Rep Ser. 1997;457:1-327.

45. Crott JW, Mashiyama ST, Ames BN, Fenech M. Folic acid deficiency increases chromosome breakage and rearrangement, gene amplification and DNA-uracil content in human lymphocytes in vitro: effect of MTHFR C677T polymorphism. Cancer Epidemiol Biom Prev 2001;10:1089-96.

46. Kimura M, Umegaki K, Higuchi M, Thomas P, Fenech M. MTHFR C677T polymorphism, folic acid and riboflavin are important determinants of genome stability in cultured human lymphocytes. J Nutrit 2004;134:48-56.
47. Fenech M, Crott, JW. Micronuclei, nucleoplasmic bridges and nuclear buds induced in folic acid deficient human lymphocytes-evidence for breakage-fusion-bridge cycles in the cytokinesisblock micronucleus assay. Mutat Res 2002;504:131-36.

48. Karaman A, Binici DN, Melikogluc MA. Comet assay and analysis of micronucleus formation in patients with rheumatoid arthritis. Mutat Res 2011;721:1-5.

49. Jia C, Jing Z, Yan W, Li QD, Chang X, Qin W et al. Cytogenetic Abnormalities in Lymphocytes from Victims Exposed to Cobalt-60 Radiation. Int. J. Mol. Sci. 2013;14: 17525-35. 